Barcelona, Spain, November 17-20, 2013

\title{
Fast Charging Method Based on Estimation of Ion Concentrations using a Reduced Order of Electrochemical Thermal Model for Lithium Ion Polymer Battery
}

\author{
Song-Yul Choe ${ }^{1}$, Xueyan Li and Meng Xiao \\ ${ }^{I}$ Song-Yul Choe (corresponding author), Mechanical Engineering, Auburn University \\ 1418 Wiggins, Auburn University, AL, 36849, USA, choeson@auburn.edu
}

\begin{abstract}
Comparably long charging time for battery of electric and hybrid vehicles is one of barriers for massive commercialization of the vehicles. Typical charging methods are by a constant current (CC) with constant voltage $(\mathrm{CV})$, pulsed or tapered current. Theoretically, the charging time can be reduced by increased amplitude of the charging current, which, however, accelerate degradation of cells and reduces the lifespan. The relationship between the charging current and the degradation has not been well understood. Studies on ion transport and chemical reactions using a computational model developed in our laboratory reveal that a high charging current causes excessive ions at the surface of electrode particles because of slow diffusion process of ions in the solid electrodes. The excessive lithium ions react with electrons and form a thin layer, called Lithium plating that is irreversible. The Lithium plating not only reduces ion conductivities, but also contributes growth of dendrites and potentially internal short circuit.

In this paper, a new charging algorithm is proposed that is based on an electrochemical and thermal model, which order is drastically reduced in order to facilitate a real time operation. The model, called Reduced Order of Electrochemical Thermal Model (ROM), is completely validated with a pouch type of Lithium polymer battery and used to dynamically estimate ion concentration at the surface of particles. Based on the estimated ion concentration, a new control algorithm is derived that allows for determination of amplitude and duration of the charging current.

The ROM performs at least ten fold faster in calculations than the original full order model. The simulation and experimental results show that the charging time can be reduced to $60-70 \%$ of that of the classical $\mathrm{CC} / \mathrm{CV}$ charging by preventing excessive ions and slowing down degradation of cell capacity losses.
\end{abstract}

Keywords: Battery Model, Fast Charge, Lithium Battery

\section{Introduction}

Battery is preferably used as rechargeable energy storage for different applications because of relative high energy and power density over costs in comparison to other storage technologies. Among the available battery technologies, the 
Lithium ion battery is the mostly accepted one because of the highest power and energy density. The areas of applications include portable electronics, hybrid and electric vehicles, renewable power systems and others, where the battery is frequently charged or discharged. Battery is charged from different power sources like a generator driven by an engine or grid or other sources. The maximum charge for a battery is predefined and accordingly the amplitude and duration should be controlled. Generally, a charger is used for supplying a constant current and voltage to reach a full charge that manufacturers specify. For other cases when a battery is charged by an inverter that is connected to electric motors, the waveform of the charging current is time variant and only amount of charge that is already stored determines the control of the current. The first charging method uses a constant current until a certain voltage and then a constant voltage to reach the full capacity, which is called $\mathrm{CC} / \mathrm{CV}$ charging. Even though this standard $\mathrm{CC} / \mathrm{CV}$ charging profile is simple to implement in battery management system, the methods have not completely optimized with respect to better performance such as larger capacity storage, larger energy storage, less charging time, less cell degradation, better safety and so on.

There have been several approaches in the past that address optimization of the charging profiles. S. Rahimian attempted to maximize cell life, charging current profile is obtained as a function of cycle number during cycling by using a dynamic optimization method [1]. R. Klein proposed an optimized charging strategy based on control theory, considering utilization constraints, maximum charging/discharging current, lower/upper cut-off voltages, operating temperature domain. So the battery can be charged fastest while guaranteeing safety throughout the battery life [2]. In addition, Y. Luo presented a Taguchi-based algorithm to determine an optimal charging pattern for multistage constant current charging method [3]. To improve battery-charge response, L. Chen proposed a variable frequency pulse charge which can detect optimal charge frequency of a battery [4]. With optimized charging methods, charging speed has been able to be improved; however, cell degradation caused by fast charging has not been taken into account. One of the significant issues is Lithium deposition that occurs in the negative electrode upon fast charge, or at low temperatures, especially at the end of charging [5],[6]. When charged, li-ions are transported from cathode to anode, which causes a high ion concentration at the surface because it takes a while for li ions to diffuse in the lattice structure and intercalate with the carbon atom structure. Lithium metal forms first near the electrode-separator boundary, where surface concentration of ions is at the highest during charging. When lithium ions cannot insert into a saturated negative electrode and plates out of the electrolyte onto the surface, leads to capacity losses of active lithium and electrolyte. It also compromises cell safety by creating the possibility for dendritic growth that leads to an internal shorting-circuiting.

The major issue for Lithium battery is a capacity fade during cycling, which is intensified by charging at high current. The reaction on the negative electrode is described as

$\mathrm{C}_{6}+x \mathrm{Li}^{+}+x \mathrm{e}^{-} \rightarrow \mathrm{Li}_{x} \mathrm{C}_{6}$

Primary side reaction taking place in anode is as follows, where the lithium ions react with electrons and form a lithium solid;

$\mathrm{Li}^{+}+\mathrm{e}^{-} \rightarrow \mathrm{Li}(\mathrm{s})$

Particularly, the side reaction above increases as the current density becomes high and the cell is overcharged. A high current leads to a high lithium surface concentration between electrolytes and the negative electrode and causes excessive ions at the surface. These excessive ions cause the side reactions and form lithium plating that eventually leads to aging and failures, particularly on the anode side. Therefore, the battery manufacturers specify the maximum charging current.

Objectives are to create a charging profile that reduces the charging time by keeping the saturation concentration that prevents from producing the lithium-plating and other side effects caused by an excessive concentration. The excessive ion concentrations can be prevented if the surface ion concentration could be measured and used to limit the charging current. Currently, there is no in-situ measurement technique that allows for direct measurements of concentrations. Therefore, a potential solution is to construct a model embedded in controller hardware and provides information of internal variables in real time.

Till to date, models being widely used by academia and industry is based on electric equivalent circuit components. However, the parameters like resistors, capacitors and voltage 
sources are not directly a function of concentrations. In addition, dependences of the model parameters on temperature are not mostly included. Therefore, the research team of Auburn University has developed a computational model based on electrochemical and thermal principle. The model is fully capable of representing two dimensional transient and non-isothermal behavior of a single cell, which is called a full order model (FOM). Electrochemical kinetics, mass and charge balances, ohmic equations and energy equations used for the model are partial differential equations (PDE). Solving the equations numerically is very computational intensive and the FOM cannot be effectively used for real world applications. Thus, the order of the FOM is reduced further to obtain a reduced order model (ROM), which runs faster and allows for integration into the battery control hardware. The ROM is a physic based model with a treatment of the PDE in the manner so as to reduce the computational time can be reduced. At the same time the model allows for estimation of state of charge (SOC) and concentrations including other internal variables.

Based on the ROM, a new fast charging method is proposed, which controls the charging current based on State-of-Charge (SOC) and the lithium concentration at the surface of electrode. The anticipated effects of this method is either protecting the battery from aging, lithium plating, capacity losses caused by the high lithium concentration during fast charging process or reducing the charging time or the combined. The charging profile produced is a form of pulse charging that consists of a combination of positive, zero and negative current. Multiple simulations and experiments show that the charging time can be reduced to $30-40 \%$ of typical CC/CV charging method.

\subsection{Principle of the Fast Charging (FC)}

Schematic diagram for the principle of the proposing new fast charging method is depicted in Figure 1. Active control of the charging current is carried out using two variables, the surface ion concentration and the SOC. Particularly, the surface ion concentration is immeasurable and thus estimated using a model that allows for estimating the concentrations based on measured current, terminal voltage and temperature. The inputs for the model are the time varying charging or discharging current, terminal voltage and ambient temperature. The outputs of the model are the surface concentration of negative electrode, SOC and terminal voltage. Due to inaccuracy of the model, a feedback controller is employed to compensate the errors of the model. The controller compares the terminal voltage of the battery with the output of the estimated terminal voltage and corrects concentrations of the models.

Once the reference values for SOC and concentration are set at a maximum allowable temperature, the charging profile can be determined based on the differences between the reference and the estimated variables from the ROM. Upon the differences, two level (charging and discharging) or three level (charging, rest and discharging) pulses are generated using a bangbang control or a Pulse-Width-Modulator (PWM) that compares the error with a triangle or saw tooth in order to create two or three states of pulse currents.

Generally, the surface concentration of negative electrode increases during charging and decreases during resting or discharging. The charging strategy consists of two parts, at the beginning, the cell is charged with a constant current rate until the surface concentration reaches an upper limitation, which is set based on physical characteristics of cell. At the second part, the cell can be rested or discharged until the surface concentration reaches a lower limitation, then constant current rate charging starts until the surface concentration reaches an upper limitation. 


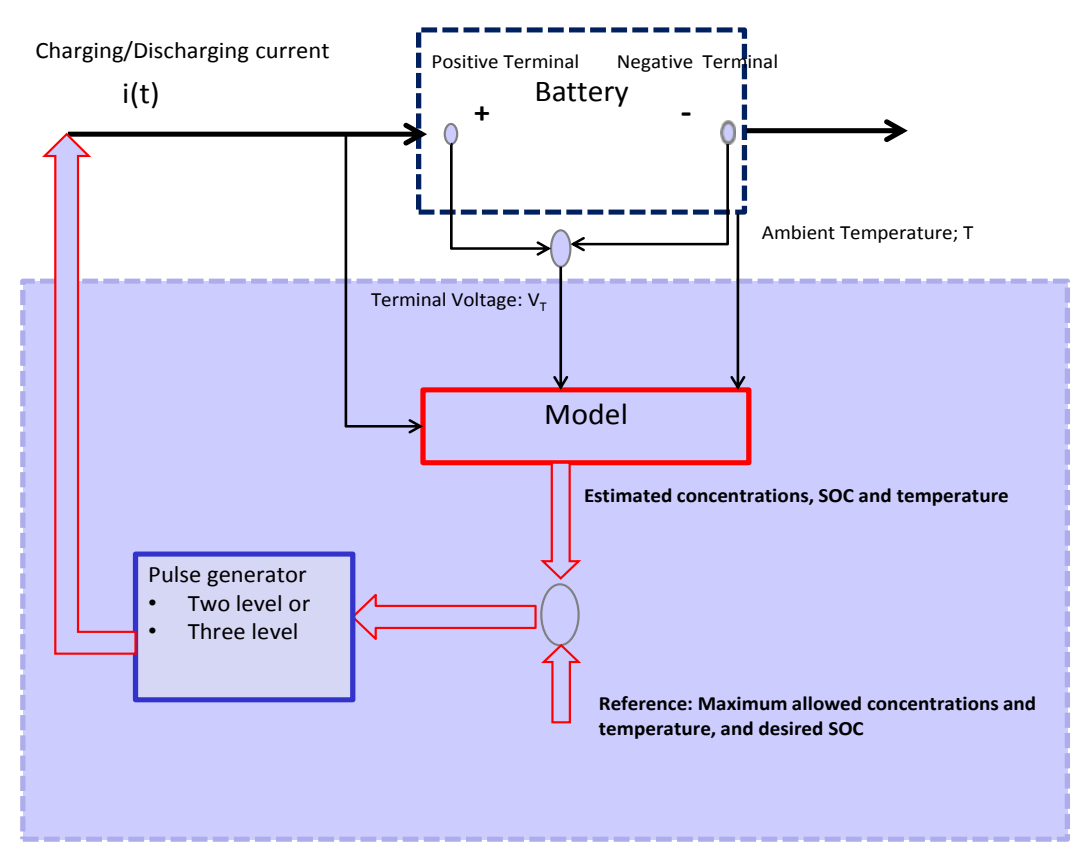

Figure 1 Schematic diagram of a new proposing charging method

\section{Reduced order model}

Models for a battery can be categorized in three different types, a model using empirical equations or electrochemical thermal principles [7] [8] or electric equivalent circuit components (ECM) [9]. Electrodes used in the electrochemical thermal model can be simplified with just one single particle model [10]. The ECM is based on open circuit voltage [11]. In addition, model errors of ECM and electrochemical thermal model are reduced by applying advanced feedback controls, such as a Kalman filter [12], linear observer [13], slidingmode observer [14], fuzzy logic [15], artificial neural network [16] and others. These methods allow for estimation of SOC and terminal voltage based on ECM, which does not consider temperature effects. In contrast, the full order of electrochemical model used for the estimation includes the thermal effects. However, the FOM has a major disadvantage of high computational time that prevents from application of the model in a real time. Therefore, the order of FOM is reduced to a model (ROM). Details on the ROM applied in this concept can be also found in the two previous papers published [17],[18]. The ROM published in the papers is improved by including a thermal behavior. There have been several authors who include effects of the temperature on the performance [18], [19]. But the heat generation caused by ion concentrations gradients is not considered. This is a crucial factor for estimation of accurate heat generation rate, especially during rest periods.

\subsection{Structure of the ROM}

A reduced order model developed consists of four sub-models; 1) Ion concentration in electrodes, 2) Ion concentration in electrolyte, 3) Potentials in both electrolyte and electrodes, and 4) Temperature in the cell.

The ion concentration in the solid electrodes is replaced with a polynomial under the assumption that ion concentrations inside the spherical particles are a function of the radius of the sphere suggested by V. Subramanian et al [21], as shown in Equation (3),

$c_{s}(r, t)=a(t)+b(t) \cdot\left(\frac{r^{2}}{R_{s}^{2}}\right)+d(t) \cdot\left(\frac{r^{4}}{R_{s}^{4}}\right)$

where the coefficients, $a(\mathrm{t}), b(\mathrm{t})$, and $d(\mathrm{t})$ are a function of time.

By combination with Fick's law and the boundary conditions, the above equations become;

$$
\begin{aligned}
& \frac{d}{d t} C_{s, a v e}+3 \frac{j^{L i}}{R_{s} a_{s} F}=0 \\
& \frac{d}{d t} q_{\text {ave }}+30 \frac{D_{s}}{R_{s}{ }^{2}} q_{\text {ave }}+\frac{45}{2} \frac{j^{L i}}{R_{s}^{2} a_{s} F}=0 \\
& 35 \frac{D_{s}}{R_{s}}\left(C_{s, \text { surf }}-C_{s, \text { ave }}\right)-8 D_{s} q_{\text {ave }}=-\frac{j^{L i}}{a_{s} F} .
\end{aligned}
$$


where the three variables are the volumeaveraged concentrations $\left(c_{s, \text { ave }}\right)$, volume-averaged concentration fluxes $\left(q_{\text {ave }}\right)$, and surface concentrations $\left(c_{s, \text { surf }}\right)$.

Hence, for ion concentration in electrolyte, distribution of ion concentrations in electrolyte and its boundary conditions is described as

$$
\begin{aligned}
& \frac{\partial\left(\varepsilon_{e} c_{e}\right)}{\partial t}=\frac{\partial}{\partial x}\left(D_{e}^{e f f} \frac{\partial}{\partial x} c_{e}\right)+\frac{1-t_{+}^{0}}{F} j^{L i} \\
& \left.\frac{\partial c_{e}}{\partial t}\right|_{x=0}=\left.\frac{\partial c_{e}}{\partial t}\right|_{x=L}=0
\end{aligned}
$$

where $C_{e}$ is the lithium-ion concentration in electrolyte phase, $\varepsilon_{e}$ is the porosity, $t_{+}{ }^{0}$ is the transference number, $D_{e}^{\text {eff }}$ is the effective diffusion coefficient calculated using the equation $D_{e}^{e f f}=D_{e} \cdot \varepsilon_{e}^{p}$.

For potentials in both electrolyte and electrode, the phase potential difference, $\phi_{\mathrm{se}}$, is defined as

$$
\phi_{s e}=\phi_{s}-\phi_{e}
$$

So, the ordinary differential equation (ODE) of the potential can be simplified as

$$
\frac{\partial}{\partial x}\left(\frac{\partial}{\partial x} \phi_{s e}\right)=j^{L i}\left(\frac{1}{\sigma^{e f f}}+\frac{1}{k^{e f f}}\right)
$$

Temperature in a battery can be simply described using the energy equation under isothermal condition,

$$
\rho C_{p} \frac{\partial T}{\partial t}=Q_{g e n}-q
$$

where $\rho, C_{p}$, and $k$ are the density, the heat capacity, and the thermal conductivity, respectively. The first term on the right describes the heat conduction per unit volume, $Q_{\text {gen }}$ is the heat generation rate per unit volume by the cell during charging and discharging and $q$ is the heat transfer rate per unit volume between the cell and its surroundings. Then, $q$ is expressed as

$q=\frac{h}{d}\left(T-T_{a m b .}\right)$

where $h, d$, and $T_{a m b}$, are the heat transfer coefficient, cell thickness, and ambient temperature, respectively.

The heat generation term is as follows that considers the heat of mixing;

$$
\begin{gathered}
Q_{\text {gen. }}=\frac{1}{L} \int_{L}\left(\Delta U_{e q u}^{+} \cdot i(r, l)-\Delta U_{e q u}^{-} \cdot i(r, l)\right) d l \\
+\frac{I}{V}\left(U_{O C V}-V_{T}-T \cdot \frac{\partial U_{O C V}}{\partial T}\right)
\end{gathered}
$$

\subsection{Effects of temperature on parameters}

Temperature changes in the cell affect chemical reactions and ion mobility. These effects are considered using the Arrhenius equation, where the diffusion coefficient representing ion transport in electrodes is expressed as a function of temperature.

$D_{s}=D_{s 0} \cdot \exp \left(\frac{E_{a}}{R}\left(\frac{1}{T_{0}}-\frac{1}{T}\right)\right)$

where $D s_{0}, E_{a}$, and $R$ are the diffusion coefficient at temperature of $T_{0}$, activation energy, and the universal gas constant, respectively. The parameters are determined by fitting experimental data to the equation 12 .

\subsection{SOC estimation}

SOC is defined as a ratio of the releasable charge capacity in a cell $\left(Q_{\text {releasable }}\right)$ to the maximum charge capacity $\left(Q_{\max }\right)$ and can be expressed by integrating the volume average charges, $\mathrm{Cs}$, ave ,in all particles from $\mathrm{L}=0$ to $\mathrm{L}_{\text {.; }}$;

$$
\begin{aligned}
& S O C=\frac{Q_{\text {releasable }}}{Q_{\max }} \\
& =\frac{1}{L_{-}} \int_{0}^{L_{-}} \frac{\left(C_{s, \text { ave }}-C_{s, \max } * \text { Stio } 0\right)}{C_{s, \max } *(\text { Stoi } 100-\text { Stoi } 0)} d x
\end{aligned}
$$

, where StoiO and Stoil00 are the stoichiometry values at $0 \%$ and $100 \%$ SOC, respectively

SOC can be directly estimated using Equation (13) where the concentration is obtained from the ROM. However, mismatch between the ROM and battery cell causes steady state and dynamic errors that can be corrected either empirically or by using a feedback loop. According to a study conducted, the empirical method has shown an immaculate accuracy during a single discharge and single cycle loading, but pulse and long-term accuracy is questionable. The error rates are not adjustable so if the dynamics of a battery differ much from those used for error rate calculations then error will grow radically. Consequently, a new SOC estimation algorithm with error correction using a feedback loop is used to remove those errors.

\section{Simulation and Validation of the ROM}

Simulation results of the reduced electrochemical thermal model are compared with experimental charging and discharging data at different current rates. The rates are $1 \mathrm{C}, 2 \mathrm{C}$, and $5 \mathrm{C}$ at $25^{\circ} \mathrm{C}$. All experimental temperatures are measured with thermocouples placed at the surface of a central 
position. A full discharge is defined as discharging from $100 \%$ SOC to $0 \%$ SOC, which corresponds to terminal voltages of $4.15 \mathrm{~V}$ at a cutoff current of $800 \mathrm{~mA}$ and $2.5 \mathrm{~V}$, respectively.

Simulated and experimental terminal voltages are compared as shown in Figure 2, where star lines represent for simulation data and solid lines for experiment data. Since thermal effect of diffusion is considered, terminal voltage response has been improved significantly, especially for high current rate at charging and discharging, where more heat is generated.
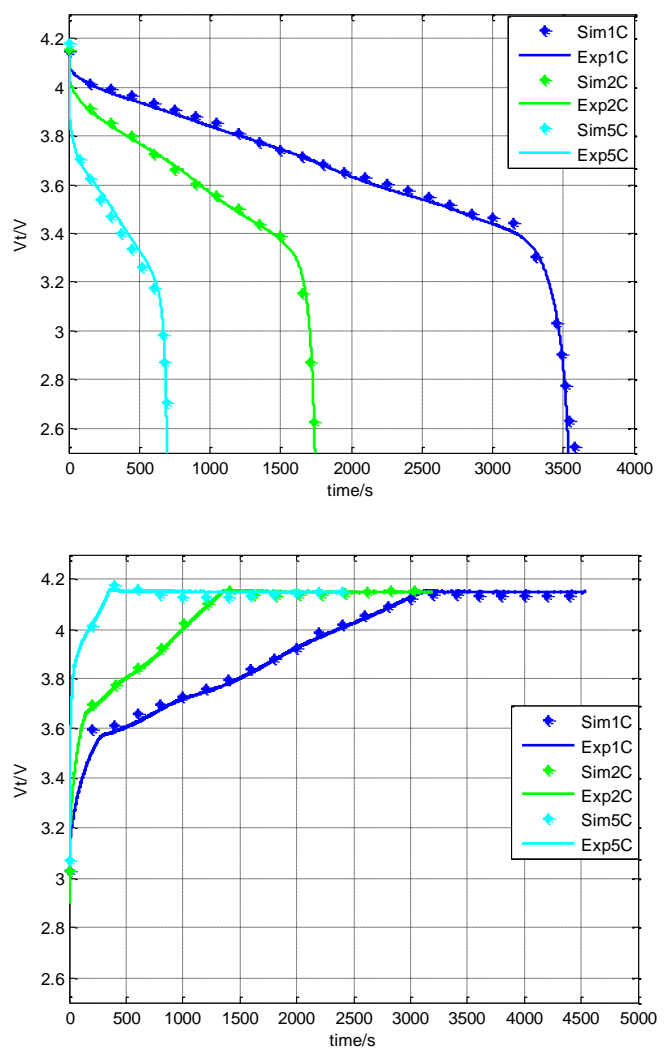

Figure 2 Terminal voltage during a full discharge and charge at different current rates.

The surface ion concentration is the variable that is used to control the amplitude and duration of the charging current. When a battery is fully charged at 5C current rate, estimated surface concentration of the solid particles is shown in Figure 3, where $\mathrm{X}$ axis represents a throughplane of a single cell that consists of a negative electrode, a separator, and a positive electrode from left to right. Each colored curve represents surface concentrations of particles at a particular time. When a charging begins, concentrations are uniform distributed in both positive and negative electrode, as shown of the red curve.
While being charged, the gradient of ion concentration increases and finally becomes zero. It should be noted that the concentration at the negative electrode-separator boundary at $500 \mathrm{~s}$ is higher than that at 2400s. In addition, due to diffusion rate limitation, the surface concentration of those particles near negative electrode-separator boundary is higher than that further away, as shown in green curve; the inverse is true for the positive electrode-separator boundary.

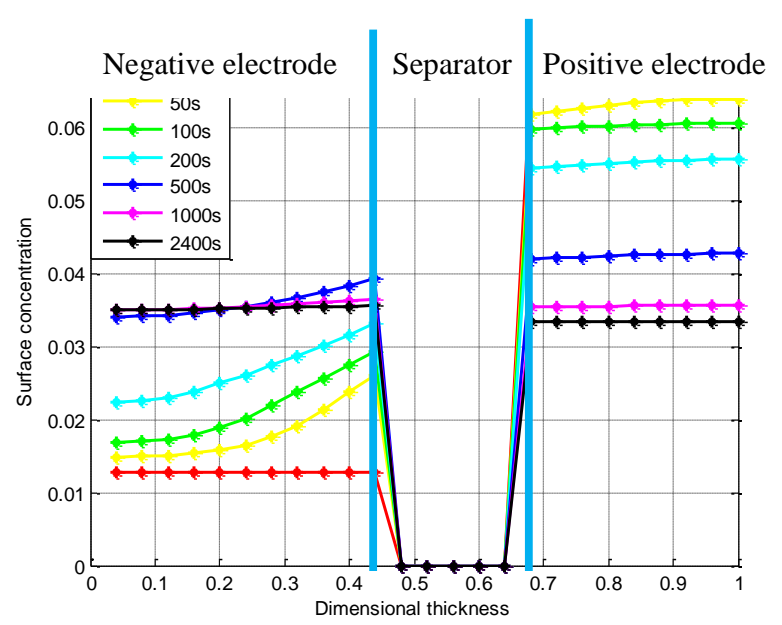

Figure 3 Surface ion concentrations of particles during a

full charge with a current rate.

The difference lasts until terminal voltage reaches $4.15 \mathrm{~V}$, and charging mode shifts to $\mathrm{CV}$ mode from $\mathrm{CC}$ mode. More details of surface concentration in time domain are shown in Figure 4, where concentration of a particle near the separator is plotted. As can be seen in the curve the concentration overshoot increases with higher charge current rates, which provides reasons of why more degradation takes place at a large current charging.

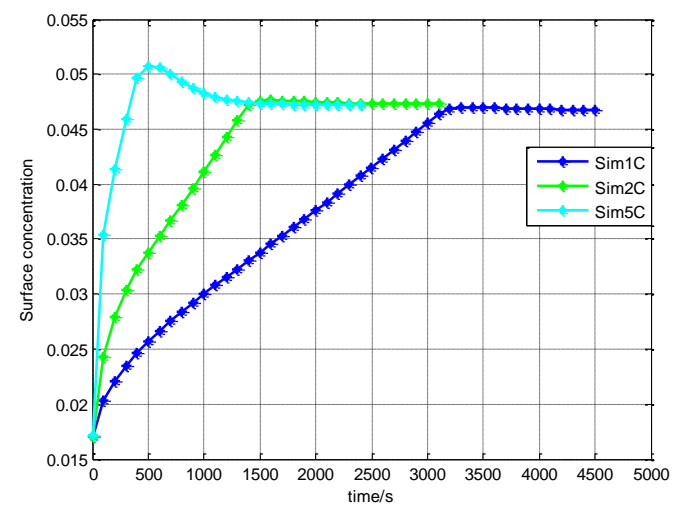

Figure 4 Surface ion concentration of particles at the boundary between negative electrode and separator during a full charge with different current rate. 
Ion concentration in electrodes is estimated as shown in 2.1. Since the sub-model for concentration is reduced in ROM compared to full order model, there will be some estimation errors. The error can be potentially minimized using a feedback control. The output of the model, including terminal voltage and temperature are compared with measured voltage and temperature, the differences are used as feedback to ROM to improve the accuracy of the model.

\subsection{Temperature}

Response of temperature at different current rates is shown in Figure 5. The estimated trend of temperature change is similar with what happens to battery. With larger discharging current, more heat is generated, and higher temperature increased is observed. In addition, simulation results were compared to experimental at 0,10 , 25 , and $40^{\circ} \mathrm{C}$. Each was a full discharge at a current rate of 1C. As shown in Figure 6, error increases as ambient temperature decreases. This is due to the $D_{s}$ curve fitting process in which ambient temperature was assumed to be constant. During low temperature discharge, the change in temperature is larger than during a high temperature discharge. These confidence intervals vary with ambient temperature and cause inaccuracy.

A ROM has been developed that includes a thermal component which simulates the temperature of a cell. Simulated results for terminal voltage more closely match those of experimental data once thermal effects were accounted for in the model. This is due to the inclusion of $D_{s}$ as a function of temperature. Previously models assume $D_{s}$ to be constant at all temperatures; however, it has been shown that $D_{s}$ will change exponentially with temperature. The diffusion coefficient, $D_{s}$, is continuously updated as a function of temperature.

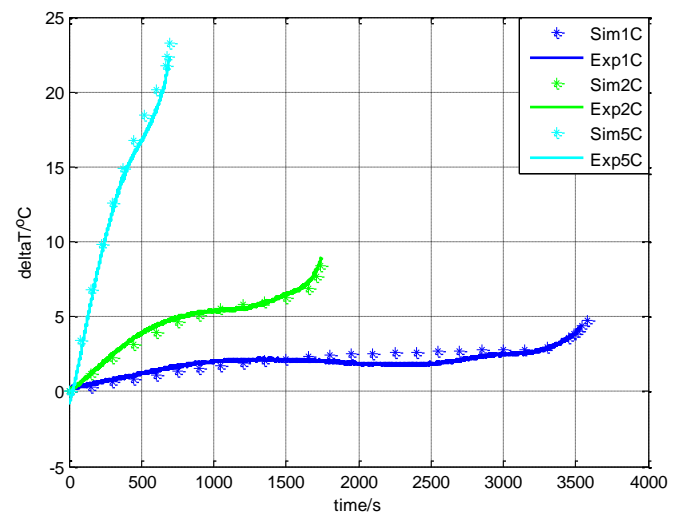

Figure 5 a) Temperature response during full discharge at different current rate. ${ }^{*}$ are simulation data and solid lines are experiment data. Blue, green, and cyan represents for $1 \mathrm{C}, 2 \mathrm{C}$, and $5 \mathrm{C}$ respectively. Ambient temperature is $25^{\circ} \mathrm{C}$.

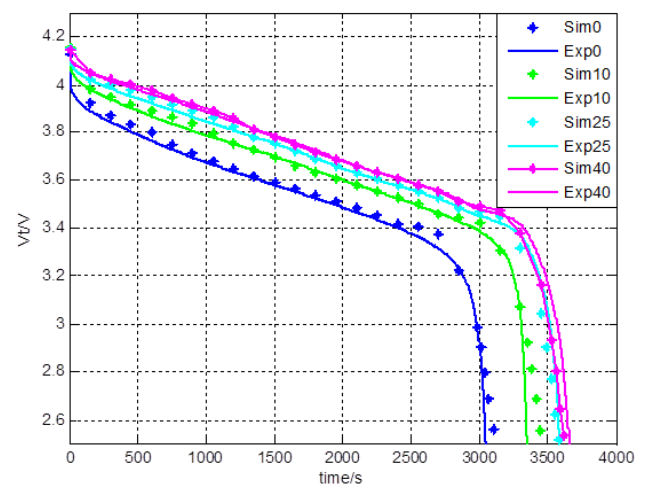

Figure 6 b) Response of terminal voltage during $1 \mathrm{C}$ full discharge at different ambient temperature. * are simulation data and solid lines are experiment data. Blue, green, cyan, and purple represents for $0^{\circ} \mathrm{C}, 10^{\circ} \mathrm{C}$, $25^{\circ} \mathrm{C}$, and $45^{\circ} \mathrm{C}$ respectively

With an increase in current comes an associated increase in heat generation rate. This explains why previous models have greater error at high current rates. The accuracy of terminal voltage and cell temperature is significantly improved compared to previous models without any thermal consideration.

Steady state error for SOC estimation error is nearly zero, when compared with the coulomb counting. Simulated temperature data closely matches experimental thermocouple data. The absolute error of the simulated temperature is less than $1.5^{\circ} \mathrm{C}$, which accurately represents temperature behavior of the cell accomplished by considering $D_{s}$ as a function of the temperature. In addition, the computational time is drastically reduced. It approximately takes $15 \%$ of the computational time of the FOM. Comparisons for both models are shown in Table 1.

Table 1 Computation time, in seconds

\begin{tabular}{|l|l|l|l|}
\hline Model & $\begin{array}{l}\text { Full } \\
\text { discharge } \\
\text { @ 1C }\end{array}$ & $\begin{array}{l}\text { Full } \\
\text { discharge } \\
\text { @ 2C }\end{array}$ & $\begin{array}{l}\text { Full } \\
\text { discharge } \\
\text { @ 5C }\end{array}$ \\
\hline $\begin{array}{l}\text { Full } \\
\text { order } \\
\text { model }\end{array}$ & 79 & 45 & 26 \\
\hline $\begin{array}{l}\text { Reduced } \\
\text { order } \\
\text { model }\end{array}$ & 8.7 & 3.9 & 2.7 \\
\hline
\end{tabular}




\section{Fast charging method}

\subsection{Simulation results}

Based on the ROM, the charging profiles are derived and simulated. The charging current is a pulse form that allows for controlling the level of ion concentrations. The pulsing current can be formed by a combination of charging, resting and discharging, which can be called a two level or a three level pulse charging.

The first simulation results of current, SOC, and surface concentration of the negative electrode at $\mathrm{CC}$ and $\mathrm{CV}$ (Red line) and the two-level pulse charging (Blue line) are shown in Figure 7. The current rate for $\mathrm{CC}$ and $\mathrm{CV}$ charging is $2 \mathrm{C}$, while the pulse charging is with $4 \mathrm{C}$. Increase of SOC at charging is shown in the second figure, where the slope of the two charging methods is different to each other. A maximum allowable concentration (green line) and saturated concentration (Blue line) is shown in the third figure, where no overshoot in the concentration is ensured, even though the pulse charging current rate is two times higher than the constant current. The charging current is controlled by the surface ion concentration and limited to a preset saturation concentration, where the charging current is turned off and discharged until the concentration drops, when the concentration exceeds the limit.
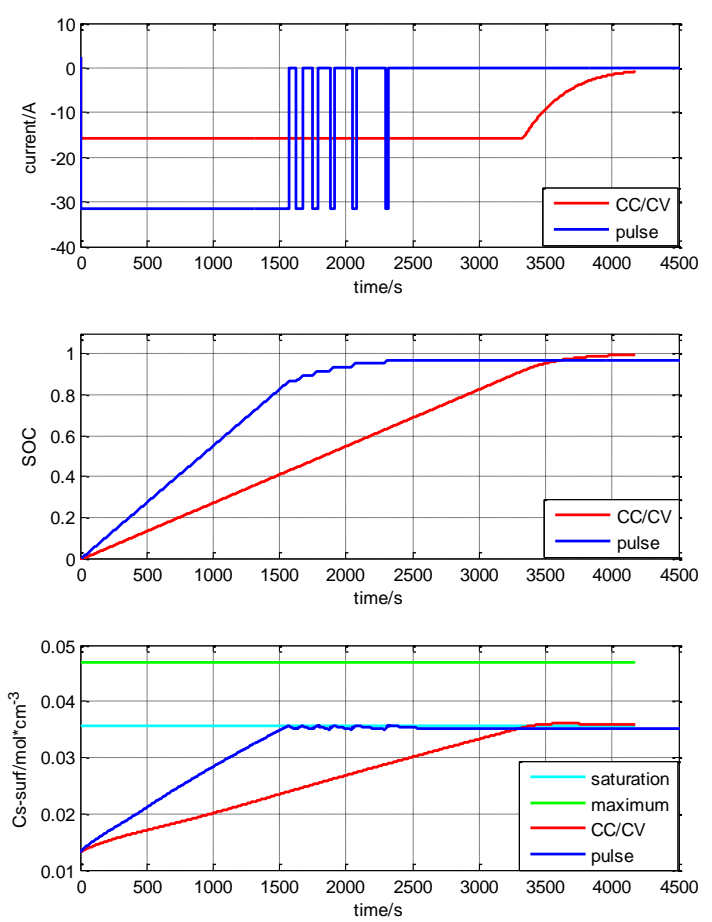

Figure 7 Simulated charging (2-level) behavior of a cell

\subsection{Experimental results with a single cycle}

The proposing new charging method is implemented in a test station and a series of tests has been conveyed. Firstly, three fresh cells are cycled at $25^{\circ} \mathrm{C}$, including full discharge at $2 \mathrm{C}$, rest, full charge, and another rest. The first cell is charged with $\mathrm{CC}$ and $\mathrm{CV}$ mode at $4 \mathrm{C}$; the second cell is charged with pulse mode at $4 \mathrm{C}$; and the third cell is charged with $\mathrm{CC}$ and $\mathrm{CV}$ mode at $2 \mathrm{C}$.

Results of a single cycle test are shown in Figure 8. Red and blue colored curves represent for pulse and $\mathrm{CC}$ and $\mathrm{CV}$ charging, respectively. Top left curve shows current. Different with CC and CV mode, the current of pulse charging is a two level charging controlled by the surface concentration. Once concentration reaches its upper limitation, the current is turned off and the cell takes a rest. However, this pulsed current causes a terminal voltage ripple that rises during the charging and decreases during discharging. In order to reduce the voltage ripples, the discharging current ripple is further reduced exponentially.

As the result shown in bottom right figure, the surface concentration that otherwise causes an overshoot shown in Figure 4 is well kept under the maximum saturation concentration, so the excessive ion concentration at the surface is also prevented. In addition, the temperature rise is less than that of $\mathrm{CC}$ and $\mathrm{CV}$ charging, which shows other advantages that degradations by the elevated temperature can be minimized. 

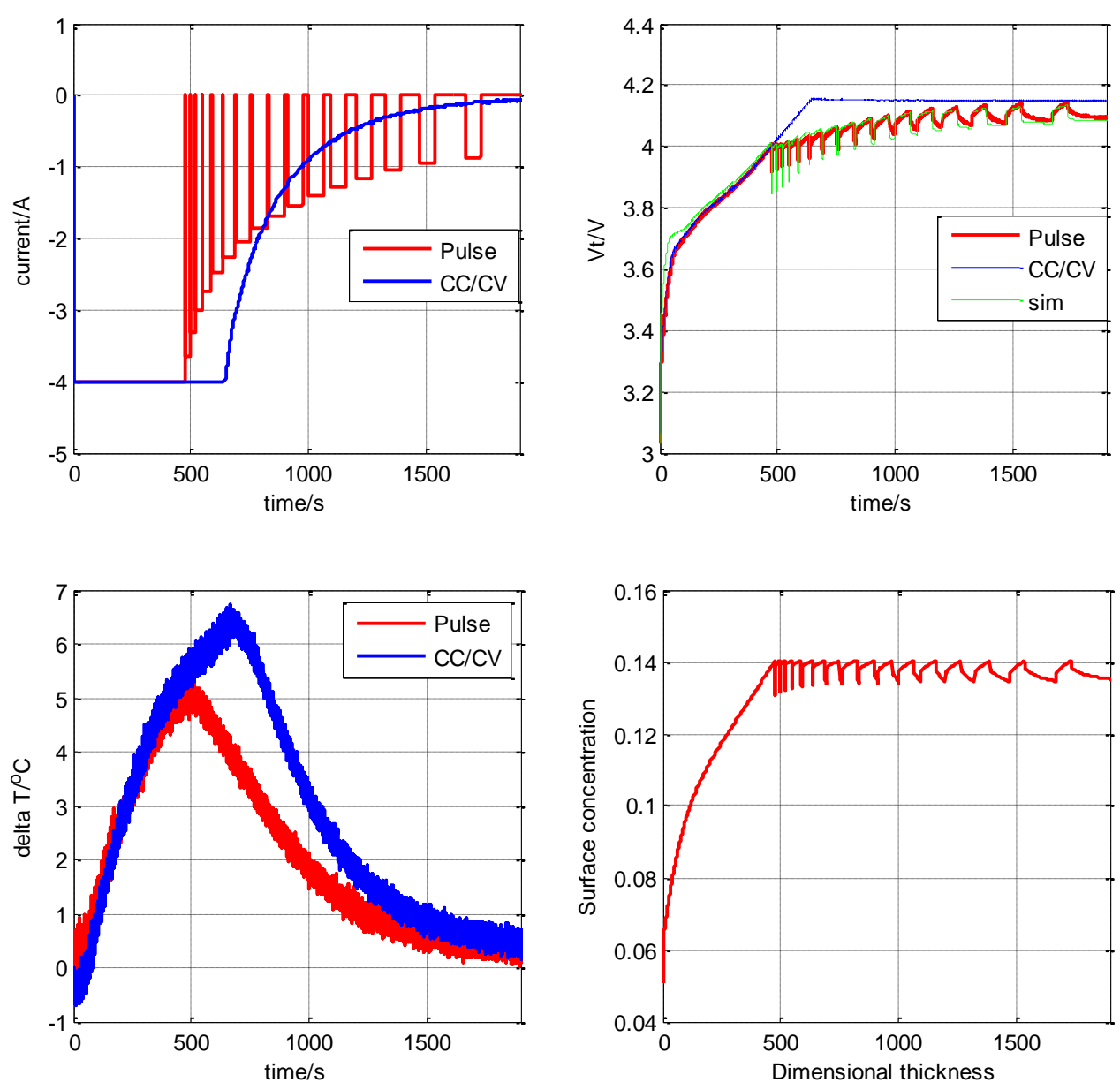

Figure 8 Comparison of experimental data between classical CC and CV charging and the proposed charging method

\subsection{Experimental results with 100 cycles for capacity losses}

A cell is tested for multiple cycles with the first and second condition described above. In order to assess cell degradation, the maximum capacity, $\mathrm{Q}_{\max }$, is measured in every 5 cycles during 100 cycles. For a measurement of $\mathrm{Q}_{\max }$, the battery is charged with in $\mathrm{CC} / \mathrm{CV}$ mode with $1 \mathrm{C}$ current rate at the first and then rested for 10 minutes. Then, $\mathrm{Q}_{\max }$ is measured during $1 \mathrm{C}$ discharge rate at $25^{\circ} \mathrm{C}$. The maximum capacity for two different charging methods is plotted in Figure 9, where $\mathrm{Q}_{\max }$ decays when the number of cycles increases. The red and blues stars represent for $\mathrm{Q}_{\max }$ with $\mathrm{CC} / \mathrm{CV}$ charging mode and the proposing charging method, respectively. It is to recognize that $\mathrm{Q}_{\max }$ of both charging method decreases linearly. However, the decay slope of the proposing method is lower than that of the classical CC and CV charging. The battery using the pulse charging has lost an amount of capacity of $0.24 \mathrm{Ah}$ after 100 cycles compared to $0.34 \mathrm{Ah}$ by the $\mathrm{CC}$ and $\mathrm{CV}$. It proves that limitation of the surface concentration of particles in the negative electrode reduces charging capacity losses caused by a relatively high charging current.

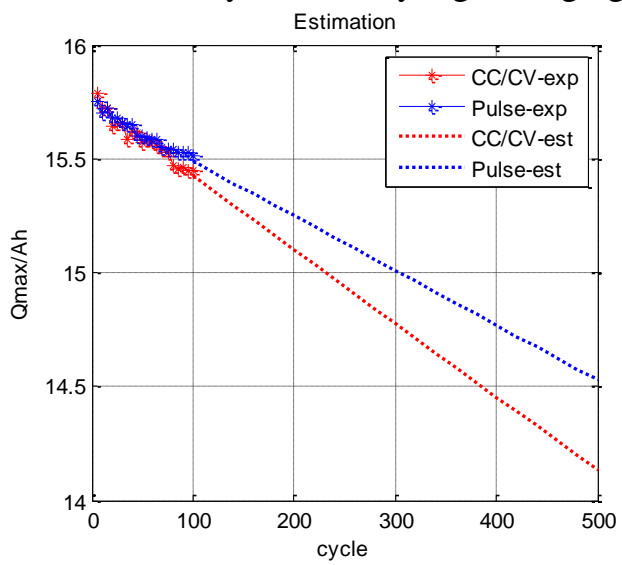

Figure 9 Comparison of Qmax between classical CC and $\mathrm{CV}$ charging and the proposed charging method 


\section{Conclusion}

A new fast charging algorithm is proposed. The algorithm is based on estimations of lithium-ion concentrations at the surface of the electrode in the anode and generates a charging current that can consist of a combination of a positive charging current, a discharging current and a zero current for resting the cell. The immeasurable surface concentrations in the electrodes are estimated using a reduced order model that is based on electrochemical thermal principles. The model is capable of being estimate not only the surface ion concentrations, but also effects of ambient temperature on the internal variables in a real time. In addition, SOC is predicted using the average concentration in the electrodes. Both the surface ion concentration and the SOC are used to control generate a profile of charging currents. Various investigations presented above show that the proposing method can either reduce the charging time in an amount of $30-40 \%$ or prevent the cell from ageing or degradation phenomena caused by excessive ion concentrations at the electrodes. The experimental data shows that the capacity losses after 100 cycles at $4 \mathrm{C}$ charging rate is $0.1 \mathrm{Ah}$ less than the conventional $\mathrm{CC}$ and CV charging.

The proposing method can be potentially used for three different applications; 1) Identical charging time with an extend lifespan, 2) Reduced charging time with the same lifetime, and 3) Reduced charging time and extended lifespan.

It should be noted that the same principle can be also applied for discharging of lithium battery as well as for charging and discharging of other battery technologies.

\section{References}

[1] S. K. Rahimian, S. C. Rayman, and R. E. White, Maximizing the Life of a LithiumIon Cell by Optimization of Charging Rates model, J. Electrochem. Soc., 157(12), pp. A1302-A1308, 2010.

[2] R. Klein, N. A. Chaturvedi, J. Christensen, J. Ahmed, R. Findeisen, and A. Kojic, Optimal Charging Strategies in Lithium-Ion Battery, 2011 American Control Conference, pp.382-387, 2011.
[3] Y.-F. Luo, Y.-H. Liu, and S.-C. Wang, Search for an optimal multistage charging pattern for lithium-ion batteries using the taguchi approach, TENCON 2009 - 2009 IEEE Region 10 Conference, pp.1-5, 2009.

[4] L. R. Chen, A design of an optimal pulse charge system by frequency-varied technique, IEEE Trans. Ind. Electron., vol. 54, pp.398-405, 2007.

[5] P. Arora, M. Doyle, and R. E. White, Mathematical modeling of the lithium deposition overcharge reaction in lithium-ion batteries using carbon-based negative electrodes, J. Electrochem. Soc., vol. 146(10), pp.3543-3553, 1999.

[6] Tang, M., P. Albertus and J. Newman, TwoDimensional Modelling of Lithium Deposition During Cell Charging, J. Electrochem. Soc. 156, pp.A390-A399, 2009.

[7] M. Xiao, S. Y. Choe, and F. Rahman, Static and dynamic analysis of Li-polymer battery using thermal electrochemical model, 2010 IEEE Conference on Innovative Technologies for an Efficient and Reliable Electricity Supply (CITRES), pp.309-316, 2010.

[8] K. Smith, C. Rahn, CY. Wang, Control oriented $1 D$ electrochemical model of lithium ion battery, Energy Conversion and Management, vol. 48, pp.2565-2578, 2007.

[9] H. He, R. Xiong, X. Zhang, F. Sun, and J. Fan, State-of-Charge Estimation of the Lithium-Ion Battery Using an Adaptive Extended Kalman Filter Based on an Improved Thevenin Model, IEEE Transactions on Vehicular Technology, vol. 60(4), pp.1461-1469, 2011.

[10] S. Santhanagopalan and R. E. White, Online estimation of the state of charge of a lithium ion cell, J. Power Sources, vol. 161(2), pp.1346-1355, 2006. 
[11] V. Pop, H. J. Bergveld, P. P. L. Regtien, J. H. G. Op het Veld, D. Danilov, and P. H. L. Notten, Battery Aging and Its Influence on the Electromotive Force, J. Electrochem. Soc., vol. 154(8), pp.A774-A750, 2007.

[12] G. L. Plett, Extended Kalman filtering for battery management systems of LiPB-based HEV battery packs. J. Power Sources, vol. 134, pp.252-292, 2004.

[13] N. A. Chaturvedi, R. Klein, J. Christensen, J. Ahmed, and A. Kojic, Modeling, estimation, and control challenges for lithium-ion batteries, 2010 American Control Conference, pp.1997-2002, 2010.

[14] I.-S. Kim, Nonlinear State of Charge Estimator for Hybrid Electric Vehicle Battery, IEEE Transactions on Power Electronics, vol. 23(4), pp.2027-2034, 2008.

[15] A. J. Salkind, C. Fennie, P. Singh, T. Atwater, and D. E. Reisner, Determination of state-of-charge and state-of-health of batteries by fuzzy logic methodology, J. Power Sources, vol. 80, pp.293-300, 1999.

[16] S. Piller, M. Perrin, and A. Jossen, Methods for state-of-charge determination and their applications, J. of Power Sources, vol. 96, pp.113-120, 2001.

[17] X. Li, M. Xiao, K. Malinowski, and S.-Y. Choe, State-of-charge (SOC) estimation based on reduced order of electrochemical model for a pouch type high power $\mathrm{Li}$ polymer battery, IEEE Vehicle Power and Propulsion Conference, 2011.

[18] X. Li, M. Xiao, and S.-Y. Choe, Reduced order of electrochemical model for a pouch type high power Li-polymer battery, International Conference on Clean Electrical Power, pp.593-599, 2011.

[19] C. r. Pals and J. Newman, Thermal Modeling of the Lithium/Polymer Battery, J. Electrochem. Soc., 142(10), pp.3274-3281,
1995.

[20] M. Guo, G. Sikha, and R. E. White, SingleParticle Model for a Lithium-Ion Cell: Thermal Behavior, J. Electrochem. Soc., 158(2), pp. A122-A132, 2011.

[21] V. Ramadesigan, V. Boovaragavan, J. Carl Pirkle, Jr., and V. R. Subramanian, Efficient Reformulation of Solid-Phase Diffusion in Physics-Based Lithium-ion Battery Models, J. Electrochem. Soc., 157, pp. A854-A860, 2010.

\section{Authors}

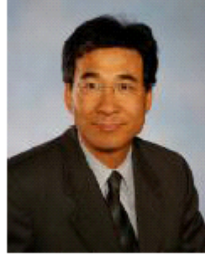

Song-Yul (Ben) Choe is a professor in the Department of Mechanical Engineering at the Auburn University, USA. His research focus is on dynamic modeling, analysis and controls of power systems and components that include hybrid systems, fuel cell, battery and high current connectors. Choe received a $\mathrm{Ph} . \mathrm{D}$ in electrical engineering from the Technical University Berlin.

Xueyan Li received the B.S. \& M.S. degree in Mechanical Engineering from Xi' an Jiaotong University. She is currently pursuing $\mathrm{PhD}$ degree in Mechanical Engineering, Auburn University, where his research efforts are focused on reduced order modelling of lithium ion battery for hybrid vehicles.

Meng Xiao received the B.S. degree in Mechanical Engineering from Tsinghua University. And he received $\mathrm{PhD}$ degree in Mechanical Engineering, Auburn University, where his research efforts were focused on modelling of lithium ion battery for hybrid vehicles in time domain and frequency domain. 